See discussions, stats, and author profiles for this publication at: https://www.researchgate.net/publication/331468649

\title{
Optimization of single crystal mirrors for ITER diagnostics
}

Article in Fusion Engineering and Design · March 2019

Dol: 10.1016/.j.fusengdes.2019.02.102

\section{CITATIONS}

3

10 authors, including:

Andrey Litnovsky

Forschungszentrum Jülich

178 PUBLICATIONS 2,509 CITATIONS

$$
\text { SEE PROFILE }
$$

2. Marcin Rasinski

Eorschungszentrum Jülich

148 PUBLICATIONS 988 CITATIONS

SEE PROFILE

\section{READS}

54

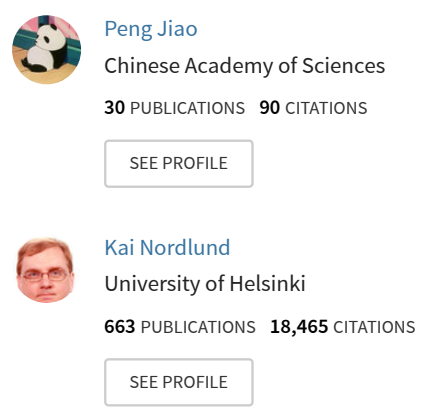

Some of the authors of this publication are also working on these related projects:

Self-passivating W alloys View project

Project Swift heavy ion tracks View project 
This is a ResearchGate version of a paper.

Please refer to the paper as follows:

A. Litnovsky, J. Peng, A. Kreter et al., „Optimization of single crystal mirrors for ITER diagnostics", Fusion Engineering and Design 146 (2019) 1450

Doi: https://doi.org/10.1016/j.fusengdes.2019.02.102

For the printed version (PDF) please contact a.litnovsky@fz-juelich.de 


\title{
Optimization of single crystal mirrors for ITER diagnostics
}

\author{
A. Litnovsky ${ }^{\mathrm{a}}$, J. Peng ${ }^{\mathrm{a}, \mathrm{b}}$, A. Kreter ${ }^{\mathrm{a}}$, Yu. Krasikov ${ }^{\mathrm{a}}$, M. Rasinski ${ }^{\mathrm{a}}$, \\ K. Nordlund ${ }^{\mathrm{c}}$, F. Granberg ${ }^{\mathrm{c}}$, J. Jussila ${ }^{\mathrm{c}}, \mathrm{U}^{\mathrm{U}}$. Breuer ${ }^{\mathrm{a}, \mathrm{d}}$ and Ch. Linsmeier ${ }^{\mathrm{a}}$ \\ ${ }^{a}$ Forschungszentrum Jülich GmbH, Institut für Energie- und Klimaforschung - Plasmaphysik, Partner of the Trilateral \\ Euregio Cluster (TEC), 52425 Jülich, Germany; \\ ${ }^{b}$ Institute of plasma physics, Chinese academy of sciences, 230031 Hefei, China; \\ ${ }^{c}$ Department of Physics, P.O. Box 43, FIN-00014 University of Helsinki, Finland; \\ ${ }^{d}$ Forschungszentrum Jülich GmbH, Central Institute for Engineering, Electronics and Analytics ZEA-3, 52425 Jülich, \\ Germany.
}

Diagnostic mirrors are planned to be used in all optical diagnostics in ITER. Degradation of mirrors due to e.g. deposition of plasma impurities will hamper the entire performance of affected diagnostics. In situ mirror cleaning by plasma sputtering is presently envisaged for the recovery of contaminated mirrors.

There are observations showing a signature of sputtering dependence on crystal orientation. Should such a dependence exist, the sputtering of single crystal mirrors could be minimized, thus prolonging a mirror lifetime.

Four single crystal molybdenum mirrors with different orientations were produced to study the effect of crystal orientation on sputtering. Mirrors were exposed to argon plasma under identical plasma conditions relevant to those expected in the mirror cleaning systems of ITER. The energy of impinging ions was about $60 \mathrm{eV}$. The amount of sputtered material corresponded to about a hundred mirror cleaning cycles in argon.

Plasma exposures did not affect the mirror reflectivity. The maximum decrease of specular reflectivity did not exceed 5\% at $250 \mathrm{~nm}$. The mirrors with orientations [110]/[101] demonstrated up to $42 \%$ less sputtering than the mirrors with other crystal orientations. These findings outline the advantage of a favorable crystal orientation for a cleaning of heavy contaminants from ITER mirrors.

Keywords: ITER diagnostic mirrors, mirror cleaning, single-crystal molybdenum, crystal orientation, sputterresistant mirrors, recovery of reflectivity.

\section{Introduction}

In the harsh particle and radiation environment of ITER, all optical and laser-based diagnostics have to observe the light from plasma via the mirror [1, 2]. A socalled diagnostic first mirror thus will become the first optical element to be exposed to plasma particles and radiation. The exposure can modify and/or contaminate the mirror surface, thus hampering the entire optical performance of the affected diagnostic. Therefore it is crucially important to keep the mirror surface clean. It is envisaged presently, to clean the ITER first mirrors in situ in the ITER diagnostics by sputtering the contaminants and the affected mirror material using cleaning plasmas [3].

Presently, the single crystal (SC) materials have proven their decisive advantages by maintaining the optical reflectivity during sputtering [4-7]. This is especially important in case of contamination with heavy impurities, like e.g. tungsten (W). In such a case, in the course of mirror cleaning the mirror itself will be sputtered easier than the contaminant, making the mirror recovery challenging. Significant deposition of $\mathrm{W}$ on diagnostic mirrors was already observed in present-day tokamaks [8]. Therefore, the resistivity to the sputtering is of significant importance. In case of single crystals, a key question is, if there is any preferential crystal orientation demonstrating the least sputtering in the conditions of plasma cleaning. There are indications that the crystal orientation does influence the sputtering resistance [9]. Finding such an orientation may lead us to maximizing the amount of sputtering cycles, thus increasing the lifetime of the first mirror in ITER diagnostics. The first dedicated sputtering test of single crystal molybdenum mirrors with different crystal orientations is the subject of this paper.

\section{Experimental}

\subsection{Rationale}

The main idea of the experiment was to expose the single crystal molybdenum (SC Mo) mirrors with all possible principle low-index crystal orientations to steady-state plasma under identical plasma conditions and to measure the sputtering. The plasma conditions should closely match the conditions expected in mirror cleaning system. For these studies argon (Ar) was used as a working gas. Argon is supposed to be used for removing heavy tungsten contaminants, should they form on the mirror surface.

\subsection{Mirror samples}

For the exposure, four SC Mo mirrors were prepared. The mirror marked SC Mo 1 had a crystal orientation [100], SC Mo 3 [110], SC Mo 5 [111] and SC Mo 7 [101] respectively. Principle orientations for a bodycentered cubic lattice system of molybdenum are described in e.g. $[10,11]$ All the mirrors were polished using the same diamond turning procedure to the final 
surface roughness $\mathrm{R}_{\mathrm{a}} \sim 1 \mathrm{~nm}$. The mirrors had a thickness of $4 \mathrm{~mm}$, and a diameter of $22 \mathrm{~mm}$. The round polished surface of the mirror had a diameter of $18 \mathrm{~mm}$.

\subsection{Pre- and post-exposure characterization}

Series of measurements were made on the mirrors before and after exposure. Total and diffuse reflectivity was measured and surface morphology and elemental composition were investigated. The surface roughness was evaluated using the stylus profiler Dektak $6 \mathrm{M}$ in the MirrorLab [12]. Scan locations are shown in Fig.1a. Each scan of surface roughness consisted of five measurements, the final result their average. Time-offlight Secondary Ion Mass-Spectrometry (ToF SIMS) investigations were made in the middle of each sample using a ToF-SIMS IV facility on locations shown in Fig 1a. SIMS measurements provided depth profiles of the elemental composition in the sample. Scanning electron microscopy (SEM) surface surveys were made on each sample on the area shown in Fig. 1a using the Carl Zeiss CrossBeam XB 540 microscope equipped with the focused ion beam (FIB). Cross-section viewing by FIB was performed in the central area of each sample shown with the smallest square in Fig.1a using special markers to directly measure the material sputtered during plasma exposure, as reported in detail in references [6, 7]. Electron Backscatter Diffraction was used for investigations of crystal orientation.

\subsection{Diagnostic arrangements}

For the exposure, the samples were installed into the sample holder with an active temperature control and mounted on the sample transfer system of the linear plasma device PSI-2 [13]. The temperature of the holder was controlled with the built - in thermocouple located behind one of the samples. The temperature of all samples during exposure was monitored with a multizone FLIR infrared camera. This camera was calibrated prior to an exposure with a thermocouple mounted in one of samples. Plasma parameters were studied with a moveable Langmuir probe system.

\subsection{Calibration experiment}

In order to study the identity of plasma conditions at the mirror locations, a so-called calibration experiment was performed. During this experiment, identical samples of polycrystalline tungsten were exposed on the locations foreseen for mirrors to argon plasma, as tuned for mirror exposure. The tungsten samples, manufactured according to ITER specifications [14], were spark-eroded from the same batch to ensure the identical surface morphology and grain structure and orientation. The exposure continued for 40 minutes, the samples were biased to $-65 \mathrm{~V}$ as the actual mirrors. The resulting ion fluence was $3.7 \times 10^{20} \mathrm{ion} / \mathrm{cm}^{2}$. The temperature during the exposure was in the range of $240^{\circ} \mathrm{C}-255^{\circ} \mathrm{C}$. After exposure, mass loss measurements were performed. The obtained data from all four test samples is provided in the Table 1 . It can be inferred that the maximum inhomogeneity of plasma parameters does not exceed 5\% which makes the entire direct comparative study feasible

Table 1 Results of uniformity tests

\begin{tabular}{|c|c|c|c|}
\hline Sample & $\begin{array}{c}\text { Averaged } \\
\text { mass } \\
\text { before } \\
\text { exposure, g }\end{array}$ & $\begin{array}{c}\text { Averaged } \\
\text { mass } \\
\text { after } \\
\text { exposure, g }\end{array}$ & $\begin{array}{c}\text { Deviation } \\
\text { from an } \\
\text { average, } \\
\% .\end{array}$ \\
\hline WAL1 & 6.86573 & 6.86374 & 2.2 \\
\hline WAL2 & 6.83152 & 6.82955 & 1.2 \\
\hline WAL3 & 6.79668 & 6.79483 & 5.0 \\
\hline WAL4 & 6.86376 & 6.86178 & 1.7 \\
\hline
\end{tabular}

\subsection{Exposures of single crystal mirrors in PSI-2}

In total, three experiments with single crystal mirrors were made. After each experiments the complete set of mirror characterization described in section 2.3 was repeated. The conditions of all three experiments were identical. During each experiment, the samples were exposed to the steady-state argon plasmas. The mirror holder was biased to $\mathrm{U}=-65 \mathrm{~V}$, the corresponding energy of impinging ions was $\sim 60 \mathrm{eV}$. The electron temperature during the exposure was $3-4 \mathrm{eV}$, plasma flux $1.35-1.5 \times 10^{17} \mathrm{ion} / \mathrm{cm}^{2}$ per second. The temperature of the mirrors during exposures was monitored to be in the range of $240^{\circ} \mathrm{C}-255^{\circ} \mathrm{C}$. A photo of the mirrors during plasma exposure is presented in Fig.1b. The total duration of the three experiments was 22 minutes and 30 seconds resulting in the accumulated averaged fluence of Ar ions of $1.9 \times 10^{20} \mathrm{ion} / \mathrm{cm}^{2}$.

\subsection{Results of sputtering studies}

As a result of three sputtering experiments, the mirrors had about $0.9-1.2 \mu \mathrm{m}$ of their material removed. SIMS measurements revealed a molybdenum mirror surface after sputtering by plasma ions free from contaminants. The results of reflectivity measurements are provided in Fig. 2. Here, the specular and diffuse reflectivity before and after sputtering experiments is plotted. As can be seen, only insignificant changes of $\mathrm{R}_{\text {spec }}$ were noticed. The maximum decrease of specular reflectivity did not exceed $5 \%$ at the wavelength of 250 $\mathrm{nm}$. The maximum change of the diffuse reflectivity $\mathrm{R}_{\text {diff }}$ was less than $1 \%$. Surface roughness $R_{a}$ remained at a low level of about $1.2 \mathrm{~nm}$.

Important information was obtained from mass loss and FIB measurements. The most important data is provided in Table 2. As it can be seen from the table, the mirrors with orientation [110]/[101] demonstrated the least sputtering after all exposures. The difference in sputtering rates reached $42 \%$. 


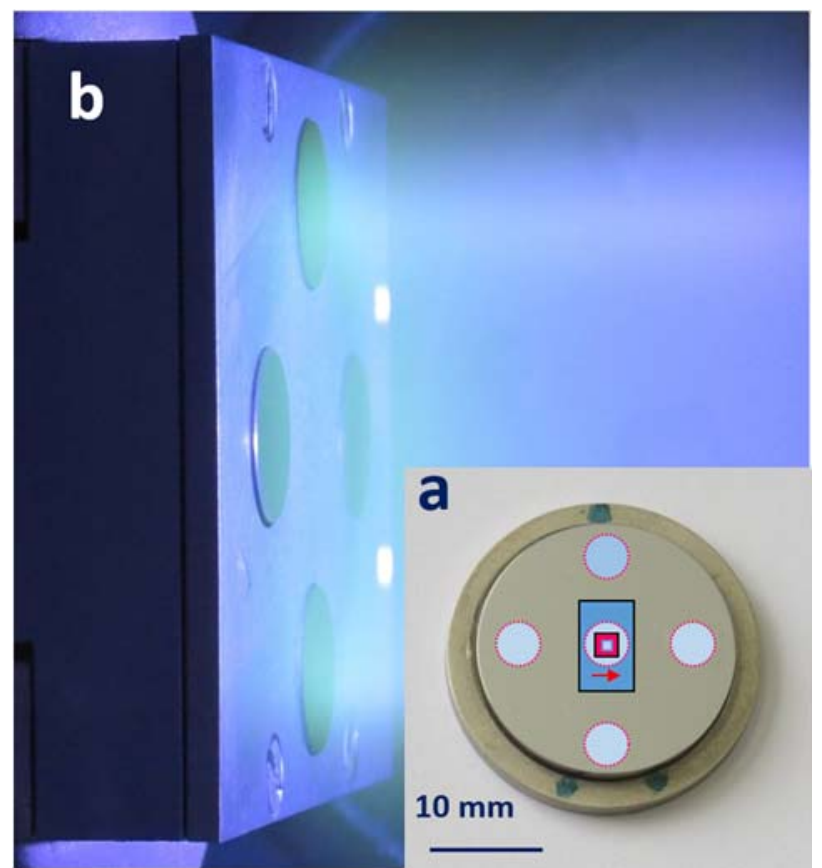

Fig.1. a) Mirror arrangement and measurement locations: round spots represent the locations of the total reflectivity $\mathrm{R}_{\text {tot }}$ measurements, the large rectangular block outlines the location of the diffuse reflectivity measurements, the largest square represents the location of the SIMS measurement, whereas the smaller square shows the location of the SEM-FIB investigations, location of surface roughness scans is shown with an arrow; b) SC Mo mirrors during exposure in PSI-2.

Table 2. Results of sputtering tests

\begin{tabular}{|c|c|c|c|c|}
\hline Mirror & $\begin{array}{c}\text { Orienta } \\
\text { tion }\end{array}$ & $\begin{array}{c}\text { Total mass } \\
\text { loss, } \mu \mathrm{g}\end{array}$ & $\begin{array}{c}\text { Deviation } \\
\text { from an } \\
\text { average, } \%\end{array}$ & $\begin{array}{c}\text { Sputtering } \\
\text { coefficient, } \\
\text { at/ion }\end{array}$ \\
\hline SC Mo 1 & {$[100]$} & 3480 & 25,3 & $1.2^{*} 10^{-1}$ \\
\hline SC Mo 3 & {$[110]$} & 2330 & $-16,3$ & $0.8^{*} 10^{-1}$ \\
\hline SC Mo 5 & {$[111]$} & 3060 & 9,9 & $1.0^{*} 10^{-1}$ \\
\hline SC Mo 7 & {$[101]$} & 2270 & $-18,5$ & $0.8^{*} 10^{-1}$ \\
\hline
\end{tabular}

\subsection{Modeling of crystal lattice effects}

To extend the understanding of the detected dependence of sputtering yield on crystal lattice orientation, molecular dynamics (MD) simulations were carried out. The simulations were performed using the PARCAS code [15-17] to obtain the sputtering yield of the differently oriented surfaces. For these studies the Mo target had the orientations identical with those in the described experiment: [100], [110] and [111]. Ar ions with a kinetic energy of $60 \mathrm{eV}$ were hitting the differently oriented surfaces perpendicularly. The ions were hitting a square box with the side length of 4 lattice constants, at $27^{\circ} \mathrm{C}(300 \mathrm{~K})$, similarly to previous studies on tungsten [18]. The interactions between Mo atoms were described by the Ackland et al. potential [19], with the addition of Ziegler-Biersack-Littmark potential by Salonen et al. [20]. Between the $\mathrm{Ar}$ and Mo atoms the potential from the DMol package [21] was used.
To obtain the sputtering yield and reflective yield of the different surfaces, 40000 ions were simulated for each surface. All ions were always hitting a pristine and thermalized surface. An atom or ion was considered sputtered/reflected, if they at the end of the simulation had a position $4.5 \AA$ above the surface. The cutoff was chosen to be larger than the cutoff of the potential, which indicates that there is no interaction anymore between the sputtered/reflected atom and the surface.
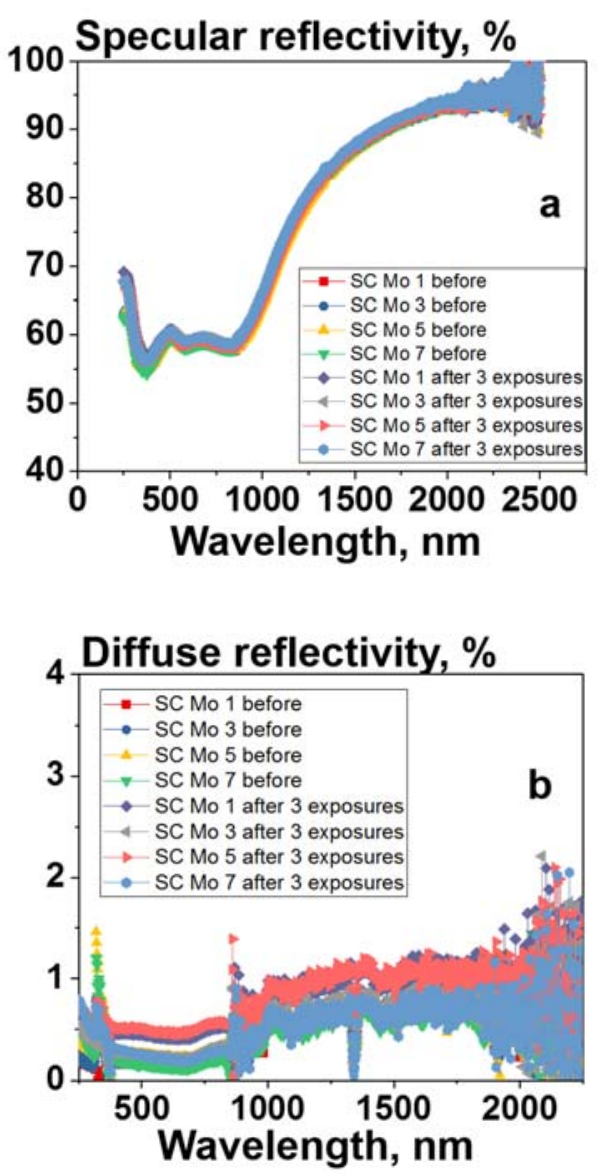

Fig.2. Dependence of a) specular reflectivity $\mathrm{R}_{\text {spec }}$ and diffuse reflectivity $\mathrm{R}_{\text {diff }}$ on a wavelength before exposure and after three exposures in cleaning argon plasma

The sputtering yield of Mo and the reflection yield of Ar of the different surfaces can be seen in Table. 3. Here we can see a clear separation between the surfaces, giving the same trend as the experiments. The [100] surface has the largest sputtering yield, as well as reflection yield. The [110] and [111] surfaces have very similar reflection yields, but the sputtering yield is completely different. The [111] surface show a sputtering yield roughly $60 \%$ of that of the [100] surface, whereas the [110] surface show almost zero sputtering yield under simulation conditions.

As can be seen, despite the qualitative agreement of modeling and experiment, the quantitative agreement was not fully attained. The possible reasons for remaining gap between experimental data and modeling is likely in more idealistic modeling 
conditions. The fully thermalized surface at temperature of $27^{\circ} \mathrm{C}$ was simulated. The ions were hitting all the time the pristine i.e. perfectly smooth surface which was obviously not valid for experimental conditions. Experiments and modeling were performed at ion energies close to the sputtering threshold of molybdenum. Under such conditions, the processes, usually dominating high-energy exposures, like ion channeling [18], are not playing a decisive role anymore. At the same time, the other processes involving e.g. adatoms may govern the sputtering. These processes can affect results significantly. Extensive modeling effort is underway, involving increasingly sophisticated surface models and allowing a better comparison with experiments. The results of this dedicated research will be reported shortly.

\section{Summary}

The effect of the crystal orientation on the sputtering coefficient of the single crystal molybdenum mirror materials was investigated. The single crystal with different crystal orientation were exposed in steadystate argon plasma under identical plasma conditions. Ion energy was $60 \mathrm{eV}$ and the sample temperature was $240^{\circ} \mathrm{C}-255^{\circ} \mathrm{C}$. Exposure parameters were relevant to those expected in the in situ mirror cleaning system currently envisaged in ITER diagnostics. In the course of three consecutive exposures the mirror samples received an argon fluence of $1.9 * 10^{19} \mathrm{ion} / \mathrm{cm}^{2}$. The resulting material sputtering was measured using the mass loss technique. Significant differences in sputtering were detected. The single crystals with the [110]/[101] orientation evidenced the least sputtering followed by the single crystal with [111] orientation and finally, the one with the [100] orientation. The difference between the most and the least sputtering resistant surface orientations reaches $42 \%$ thus exhibiting sound advantages of the favorable crystal orientation in prolonging the lifetime of diagnostic mirrors under sputtering conditions.

Table 3. Sputtering and reflection yields from $M D$

\begin{tabular}{|c|c|c|}
\hline Orientation & $\begin{array}{c}\text { Sputtering yield, } \\
\text { at/ion }\end{array}$ & $\begin{array}{c}\text { Reflection yield, } \\
\text { at/ion }\end{array}$ \\
\hline$[100]$ & $4.6 * 10^{-2}$ & 0.90 \\
\hline$[110]$ & $0.048 * 10^{-2}$ & 0.83 \\
\hline$[111]$ & $2.7 * 10^{-2}$ & 0.83 \\
\hline
\end{tabular}

First modeling estimates were done using molecular dynamics. Experimental conditions: geometry, ion energy and crystal orientations were used for modeling. The first results show qualitatively the same trend as the experiments, where the same order in sputtering yield was reproduced, which indicates the viability of this method in further studies. The discrepancy in exact values is most likely due to more idealistic conditions used in modeling where the monoenergetic ions were bombarding the perfectly smooth molybdenum surface.

\section{Outlook}

Future investigations will be focused on improving modeling algorithms followed by new estimates of sputtering of single crystals with different crystal orientation - aiming at gaining the better understanding of underlying physics processes. The modeling estimates will be followed by experimental studies addressing the effect of crystal orientation on sputtering of single crystal rhodium mirrors.

\section{Acknowledgments}

The authors would like to thank Dr. P. Shigin and Dr. R. Reichle from ITER Organization for helpful advices and support. The authors are grateful to S. Kraus and M. Vogel from FZJ for the technical assistance and help in preparation and conduction of the experiment. Experimental activity is partially supported via ITER purchase order Nr. 4100006576, IDM UID: U9F2VW. This work has been also partly carried out within the framework of the EUROfusion Consortium and has also received partial funding from the Euratom research and training programme 2014-2018 under grant agreement no. 633053. Grants of computer time from the Finnish Grid and Cloud Infrastructure (persistent identifier urn:nbn:fi:research-infras-2016072533) are gratefully acknowledged.

\section{Disclaimer}

The views and opinions expressed herein do not necessarily reflect those of the European Commission or ITER Organization.

\section{References:}

[1]. V.S. Voitsenya, A.E. Costley, V. Bandourko et al., Rev. Sci. Instrum. 72, (2001) 475;

[2]. A. Litnovsky, V. Voitsenya, T. Sugie et al., Nucl. Fusion 49 (2009) 075014;

[3]. F. Leipold, R. Reichle, V. Vorpahl et al., Rev. Sci. Instr. 87 (2016) 11D439

[4]. V. Voitsenya, A. Bardamid, V. Bondarenko et al., J. Nucl. Mater 290 (2001) 336;

[5]. A.Litnovsky, G. De Temmerman, K. Yu. Vukolov et al., Fus. Eng. and Design 82 (2007) 123;

[6]. A. Litnovsky, Yu. Krasikov, M. Rasinski et al., Fus. Eng. and Design 123 (2017) 674;

[7]. J. Peng, A. Litnovsky, A.Kreter et al., Fus. Eng. and Design 128 (2018) 107;

[8]. A. Litnovsky, M. Matveeva, A. Herrmann et al, Nucl. Fusion 53 (2013) 073033;

[9]. M.Rasinski, A. Kreter, Y. Torikai and Ch. Linsmeier, N. Mater. and Energy 12 (2017)302;

[10]. "Crystal orientation manual" by Elisabeth A. Wood, Columbia University Press (1963), New York, ASIN B0006AY5IM.

[11]. "Lecture notes on solid state physics" by Professor Steven H. Simon, Oxford University, available at: https://wwwthphys.physics.ox.ac.uk/people/SteveSimon/condmat201 2/LectureNotes2012.pdf 
[12]. MirrorLab Website: https://tec.ipp.kfajuelich.de/mirrorlab/, Access details: mirrorlab@fzjuelich.de;

[13]. A.Kreter, C. Brandt, A. Huber et al., Fusion Sci. Technol. 68(2015)8;

[14]. ITER Tungsten Bar Procurement Specifications, ITER Document: ITER_D_2X38PN.Y;

[15]. K. Nordlund, M. Ghaly, R.S. Averback, M. Caturla, T. Diaz de la Rubia, J. Tarus Phys. Rev. B, 57 (1998), pp. 7556-7570,

[16]. M. Ghaly, K. Nordlund, R.S. Averback Philos. Mag. A, 79 (1999), pp. 795-820,

[17]. K. Nordlund Comput. Mater. Sci., 3 (1995), pp. 448-456

[18]. J. Jussila, F. Granberg, K. Nordlund, Nucl. Mater. Energy, 17 (2018) 113,

[19]. G. J. Ackland, R. Thetford, Phil. Mag. A, 56 (1987) 15

[20]. E. Salonen, T. Järvi, K. Nordlund, J. Keinonen, J. Phys. Cond. Matt. 15 (2003) 5845-5855

[21]. K. Nordlund, N. Runeberg, D. Sundholm, Nucl. Instr. Meth. Phys. Res. B., 132 (1997) 45-54 